\title{
Coordinated and Aerobic Exercise do not Improve Attention in Graduate Students
}

\author{
Elizabeth K. Bailey $^{1, *}$, T.J. Douglas ${ }^{2}$, Dana Wolff $^{2}$ and Stephen Bailey ${ }^{3}$ \\ ${ }^{I}$ Department of Health and Human Performance, Elon Univerity, Elon, NC, USA \\ ${ }^{2}$ Department of Exercise Science, Elon University, Elon, NC, USA \\ ${ }^{3}$ Department of Physical Therapy Education, Elon University, Elon, NC, USA
}

\begin{abstract}
Acute coordinative exercise, represented by various sports skills requiring bilateral use of hands or feet, has been shown to improve attention in school age children in a classroom setting. The purpose of this investigation was to determine if acute aerobic and coordinative exercise improves attention in graduate students in the same setting. Twentyeight students ( 19 Women, 9 men; Age $=24 \pm 1$ years; $B M I=22.9 \pm 0.6$ ) enrolled in a graduate education program completed 3 sessions, each separated by $7 \mathrm{~d}$. Immediately before a $1 \mathrm{~h}$ classroom lecture, subjects completed either $15 \mathrm{~m}$ of quiet sitting, aerobic exercise (walking), or coordinative exercise. Coordinative exercise consisted of a sequence of bilateral activities requiring gross and fine motor movement using balls of various sizes and types. Prior to and immediately after the lecture, subjects completed the $\mathrm{d} 2$ Test of attention. Subjects did not exercise or drink caffeine prior to participation. Heart rate was similar during aerobic and coordinative exercise. The total number of items processed (TN) and concentration performance $(\mathrm{CP})$ increased from immediately post exercise to post-lecture in all conditions. The number of errors following coordinative exercise before the lecture was greater than the other conditions. The results of this investigation suggest that aerobic and coordinative exercise do not influence attention in graduate students.
\end{abstract}

Keywords: Aerobic, classroom processing, cognition, coordination.

\section{INTRODUCTION}

The effect of physical activity on cognitive function has generated strong interest recently. Acute bouts of aerobic exercise have been found to positively affect cognitive function in various age groups [1-3]. A review of studies done with adults provides strong support for the role of submaximal aerobic exercise of $60 \mathrm{~min}$ or less in facilitating aspects of information processing and cognitive function [4], while a meta-analysis of data from studies with school age children (4-18 years) suggests a positive relationship between physical activity and cognitive performance in a number of specific categories [5]. The exact mechanisms underlying the positive impact of acute exercise on cognitive function remain unclear; however, it has been hypothesized that they could be associated with changes in metabolism in the brain, increased cerebral blood flow, increased arousal, and improved relaxation [1].

In an academic setting the potential impact of acute exercise on attention is of particular interest. It is a commonly held belief that students do not pay attention

*Address correspondence to this author at the Department of Health \& Human Performance, Elon University, Elon, NC 27244, USA; Tel: +1(336) 278-5862; Fax: +1(336) 278-4143; E-mail: ebailey @elon.edu during an entire classroom period and some authors suggest that an unmotivated student is unlikely to pay attention beyond $20 \mathrm{~m} \mathrm{[6]}$. Work by Bunce and colleagues [7] indicate that college-aged students experience ever-shortening cycles of engagement $v s$. non- engagement during a traditional 50 $\mathrm{m}$ lecture as well.

While most of the previous work examining the impact of exercise on cognitive function has focused on aerobic exercise, it is possible that other types of activities may be even more beneficial in an acute setting. Specifically, coordinative exercise, represented by various sports skills requiring bilateral use of hands or feet, may be particularly beneficial because of its ability to engage specific brain areas (cerebellum and frontal lobe) that have been associated with attention $[8,9]$. This phenomenon has been demonstrated in adolescents by Budde and colleagues [10] who found that coordinative exercise had a more profound effect than traditional moderate exercise on attention using the $\mathrm{d} 2$ Test, a letter cancellation test measuring elements of sustained and selective attention. It is unclear if the positive impact of coordinative exercise extends beyond this age group. Consequently, the purpose of this investigation was to compare the effects of coordinative and aerobic exercise on attention in graduate students. 


\section{METHODS}

\section{Participants}

Twenty-eight students ( 19 Women, 9 men; Age $=24 \pm 1$ years; BMI $=22.9 \pm 0.6$ ) enrolled in a Doctor of Physical Therapy Education program served as subjects in this investigation. Sixty four percent of subjects met or exceeded the American College of Sports Medicine's recommendations for aerobic exercise [11]. Subjects completed 3 experimental sessions, each separated by $7 \mathrm{~d}$. Prior to the initiation of this study, each subject signed an informed consent approved by the Elon University Institutional Review Board for Protection of Human Subjects in Research. Subjects were asked to abstain from consuming caffeine and exercise for $3 \mathrm{~h}$ prior to each experimental session.

\section{Experimental Design}

During this investigation, subjects completed each of three experimental conditions: rest (REST), aerobic exercise (AERO), or coordinated exercise (COORD). After each experimental condition, subjects participated in a $1 \mathrm{~h}$ classroom lecture period. Attention of subjects was evaluated at two time points: 1) immediately after the exercise intervention and before the lecture (IPE) and 2) after the lecture (Post Lecture). All lectures revolved around the same general topic (research methods) and took place between 3 and $4 \mathrm{pm}$. Experimental conditions (REST, AERO, and COORD) were applied in a randomized counterbalanced fashion to control for any order effect and so that the specific lecture material and other academic expectations could be controlled.

\section{Experimental Conditions}

REST. During REST subjects completed a $15 \mathrm{~m}$ nonactive control session where they sat quietly at their desks. They were not allowed to participate in any academic activity, to access any electronic devices, or communicate with others during this time.

AERO. During AERO subjects walked laps inside the academic building for $15 \mathrm{~m}$. Subjects were encouraged to walk at a moderate pace ( 4 to 6 on the 10 point Borg RPE Scale) and not to talk to others during their AERO session. Subjects wore a Polar Heart Rate monitor during AERO and heart rate was recorded at 5, 10, and 15 minutes of AERO. For each subject, the three heart rate measures were averaged and so one heart rate measure is reported for each subject.

COORD. During COORD subjects completed a series of bilateral activities requiring gross and fine motor movement using balls of various sizes and types similar to those described by Budde and colleagues [10]. Subjects completed 5 different coordinated activities in a rotating station format. Subjects completed each station in groups of 6 , spent $2 \mathrm{~m}$ at each station, and had $1 \mathrm{~m}$ to transition between each station. Activities at each station were: Station 1) subjects bounced volleyball with alternative hands while standing on a bench; Station 2) subjects bounced a volleyball and basketball simultaneously while standing; Station 3) subjects threw a tennis ball with alternating right and left hands into a basket at a distance of 10 meters. During this task subjects were provided a large number of tennis balls in an easily accessible basket so that subjects did not need to seek balls to complete the task; Station 4) two subjects faced each other at a distance of 5 meters and simultaneously passed a tennis ball (by alternative hands) and a soccer ball (by alternative feet) back and forth to each other; Station 5) subjects dribbled a basketball with their hands and a soccer ball with their feet while moving back and forth over a 30 meter tiled surface. Subjects wore a Polar Heart Rate monitor during COORD and heart rate was recorded at 5, 10, and 15 minutes of COORD. For each subject, the three heart rate measures were averaged and so one heart rate measure is reported for each subject.

\section{Attention}

Attention was assessed using the $\mathrm{d} 2$ Test for attention in a group, in a pencil and paper format [12]. All subjects completed the test simultaneously under the supervision of the same proctor. Subjects were given the test worksheet facing down on their desk. Prior to administration, the specific directions for the test were read to subjects and provided in a written form. After being provided test directions, subjects were instructed to turn over the work sheet and begin the test at the same time. During the test the subject scans a series of letters (d or $p$ ) that each have 1-4 dashes ( 2 above or 2 below) around each letter. Subjects are instructed to only cross out the letter "d" with two dashed lines. Worksheets have 14 lines each with 47 characters for a total of 658 items. Subjects view each line of text separately for 20 seconds. The d2 Test measures processing speed, rule compliance, and quality of performance. Performance on the test is determined by evaluating the total number (TN) of items processed, the number of mistakes or errors made during the test, and by calculating concentration performance (CP) [12]. TN accounts for all of the items processed, both relevant and irrelevant, and is representative of attention allocation, processing speed, amount of work completed, and motivation. The number or errors that occurred during the test includes errors of omission and commission. It is representative of the accuracy of work. $\mathrm{CP}$ is calculated by subtracting the number of errors of commission from the number of correctly crossed out items during the test. CP is an index of the integration of speed and accuracy during the test.

\section{STATISTICAL ANALYSIS}

Differences in measures of attention (TN, CP) between conditions and across time were analyzed using a Multiple Analysis of Variance (MANOVA). When differences between conditions or across time were observed, specific differences between conditions were evaluated using the Tukey post-hoc test. Differences in the heart rate response to AERO and COORD were assessed using a paired t-test. All data are presented as mean \pm standard error $(\mathrm{M} \pm \mathrm{SE})$. Data were analyzed using the IBM Statistics package software version 21.0 (IBM Statistics, Armonk, NY, USA). 


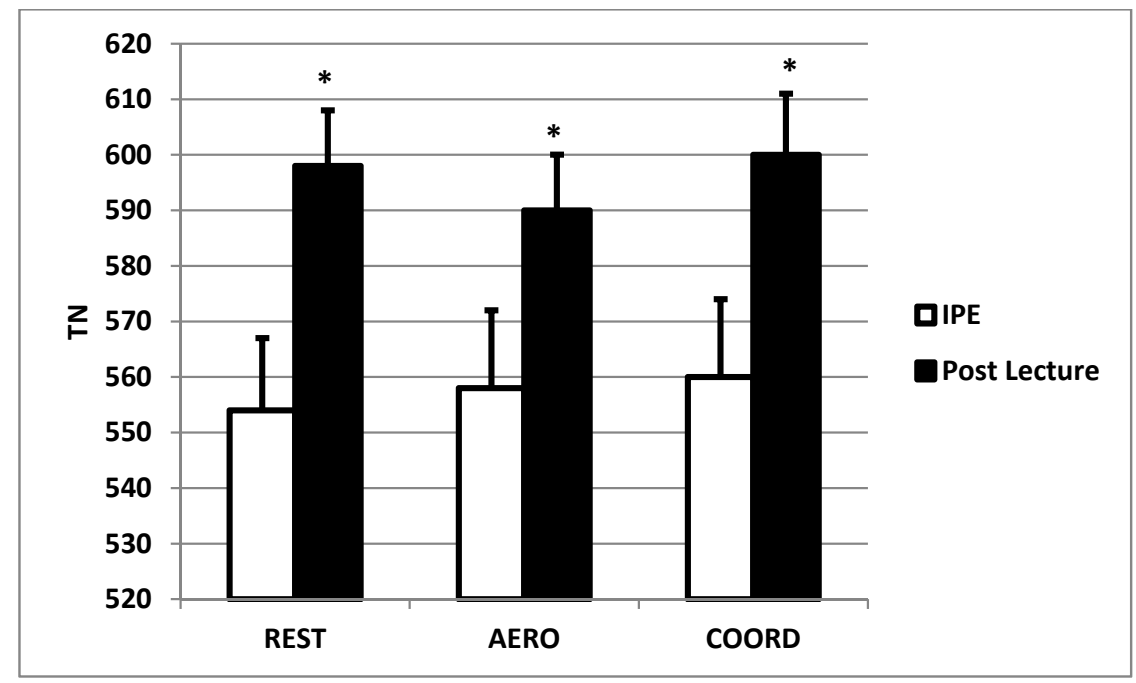

*Indicates difference from Pre $(\mathrm{p}<0.001)$

Fig. (1). Impact of aerobic (AERO) and coordinated exercise (COORD) on the total number (TN) of items processed during the d 2 Test.

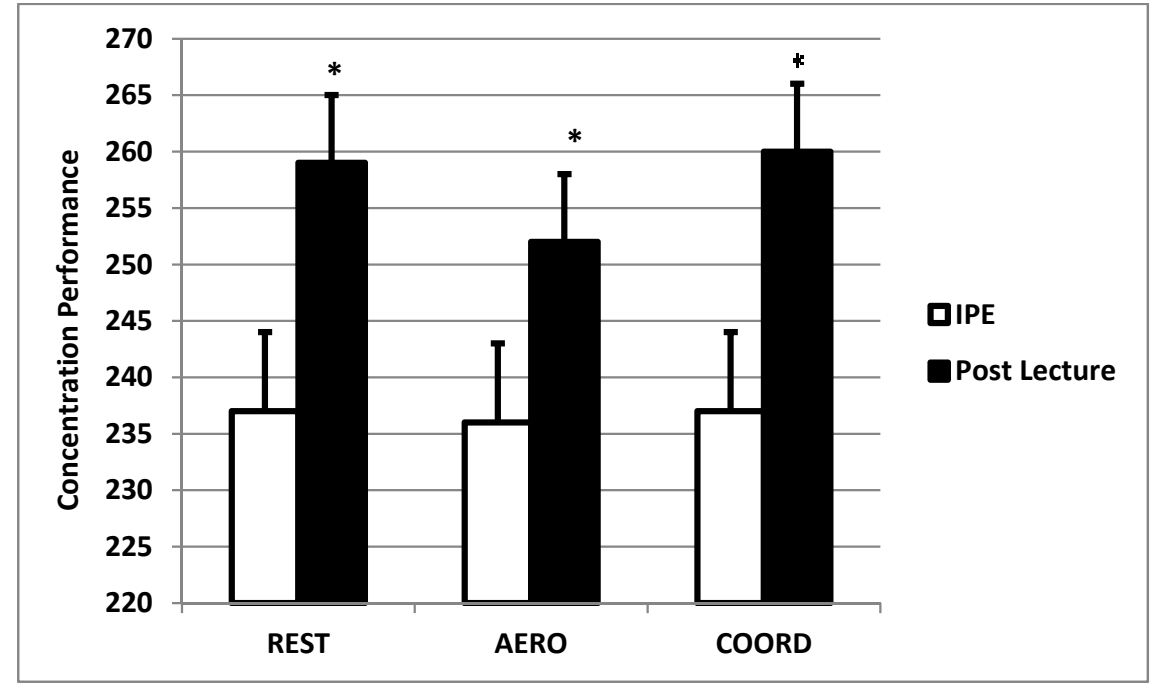

*Indicates difference from Pre $(\mathrm{p}<0.001)$

Fig. (2). Impact of aerobic (AERO) and coordinated exercise (COORD) on concentration performance during the d2 Test.

\section{RESULTS}

Heart rate was similar during AERO (121 $\pm 3 \mathrm{bpm} ; 62 \%$ of predicted maximal heart rate) and COORD (125 $\pm 4 \mathrm{bpm}$; $64 \%$ of predicted maximal heart rate) experimental sessions. These heart rate responses indicate that subjects exercised at a moderate intensity during AERO and COORD [11]. The total number $(\mathrm{TN})\left[\mathrm{F}(1,87)=108.07, p<0.001, \eta^{2}=.554\right]$ of items processed and concentration performance (CP) $\left[\mathrm{F}(1,87)=104.07, p<0.001, \eta^{2}=.545\right]$ increased from IPE to Post Lecture in all conditions (Figs. 1 and 2). There was no difference between conditions in either of these measures at any time point. An interaction between time and experimental condition was observed in the number of errors committed by subjects during the test $[\mathrm{F}(2,87)=3.54$, $\left.p=0.033, \eta^{2}=.075\right]$. Post-hoc analysis revealed that the number of errors was higher before the lecture (IPE) during
COORD compared to both REST and AERO ( $\mathrm{p}=0.040)$ (Fig. 3). The number of errors after COORD (Post Lecture) was lower than before the lecture $(\mathrm{p}=0.035)$, but it was not different from the number of errors Post Lecture during REST and AERO.

\section{DISCUSSION}

It is generally accepted that students have difficulty maintaining attention in a traditional classroom setting. The purpose of this investigation was to determine if acute aerobic or coordinative exercise could be useful tools in assisting students to maintain attention in the classroom. Unfortunately, none of the components of the $\mathrm{d} 2$ Test used to evaluate attention in this investigation were influenced by aerobic or coordinative exercise. 


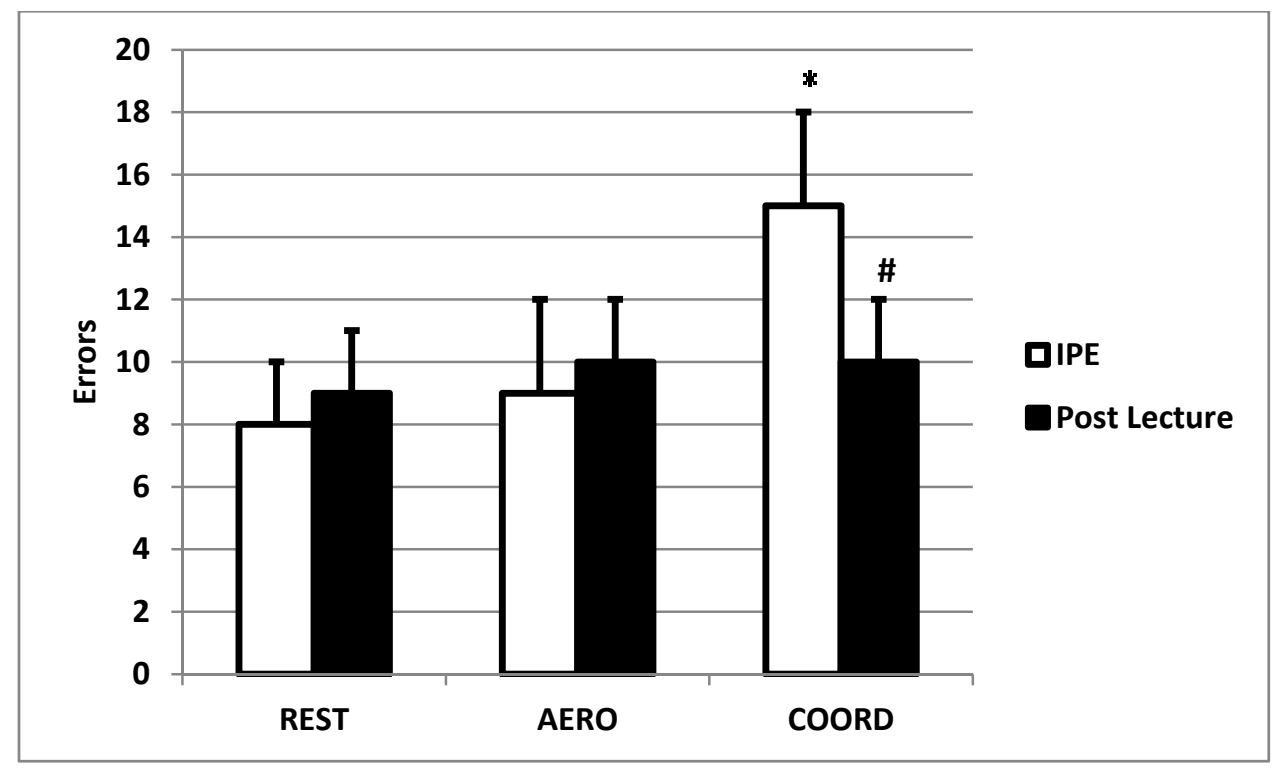

*Indicates difference from Pre REST and Pre AERO $(\mathrm{p}<0.040)$.

\# Indicated difference from Pre COORD $(\mathrm{p}<0.035)$.

Fig. (3). Impact of aerobic (AERO) and coordinated exercise (COORD) on total errors (omission and commission) during the d2 Test.

The findings of this investigation differ from those provided by Budde $e t$ al. in adolescents [10], Budde et al. in adult students [13], Gallotta et al. in 8 to 11 yo [14], and Palmer, Miller \& Robinson in preschoolers [15]. Our investigation is most comparable to that of Budde and colleagues (10), in that we used the same tool to evaluate attention ( $\mathrm{d} 2$ Test) and we patterned our COORD intervention after the one described by these authors. Budde and colleagues [10] found COORD to improve attention with better performance on the $\mathrm{d} 2$ Test following it than after a resting control condition. It is possible that the differences in response seen between these two investigations could be due to the age and maturity of participants. It could also be possible that the intensity of the intervention could have influenced the different outcomes. This is unlikely because the heart rate response to the activity was relatively higher in this investigation ( $64 \%$ of predicted maximal HR) than in Budde et al. [10] ( $\sim 59 \%$ of predicted maximal HR). Another possibility is that the way subjects perceived the activity may have influenced their attention. Perhaps the adolescents had a more positive view of the activity than the graduate students in this investigation. Neither investigation examined this dimension so any discussion on this issue would be purely speculative.

Very little information exists describing the impact of moderate aerobic activity on attention as assessed by the $\mathrm{d} 2$ Test. Budde and colleagues [13] have examined the impact of maximal aerobic exercise in adult students (ages 19-29) on performance on the $\mathrm{d} 2$ Test and concluded that highly physically active participants ( $>3$ times a week at the levels recommended by the American College of Sports Medicine) had a positive improvement in attention, while less active participants ( $<3$ times a week) saw no benefit. This different response to aerobic activity as compared to our investigation could be easily explained by exercise intensity, suggesting that a relatively high exercise intensity is needed for aerobic exercise to have an impact on attention as measured via the d2 Test.

Gallotta and colleagues [14] also describe an improvement in performance in the $\mathrm{d} 2$ Test after physical activity; however, the findings of this study are confounding in that they also saw an improvement in attention after classroom instruction. These authors argue that the stress or stimulus of the activities is the underlying mechanism of these improvements and the results are not due to a learning effect. Examination of the data presented here and the data presented by Budde et al. [10] and by Gallotta et al. [14] suggest that performance on the d2 Test is significantly impacted by repeated exposure. We attempted to control for this by counterbalancing the conditions applied to subjects. This approach may have been successful at controlling the impact of an order effect on our findings; however, it may have introduced so much error into the data that any potential impact of the exercise interventions was not apparent.

Palmer, Miller, and Robinson [15] found attention to be improved in preschoolers after a planned $30 \mathrm{~m}$ movement program as compared to a sedentary control condition. The authors applied the Picture Depletion Task for Preschoolers (DPTP) and found an approximately $42 \%$ reduction in the number or errors of omission. In comparison, the improvements described by authors using the $\mathrm{d} 2$ Test are at best $15 \%$. When considering the impact of the learning effect on the $\mathrm{d} 2$ Test and the comparatively low magnitude of the response in outcome measures, it seems reasonable to conclude the $\mathrm{d} 2$ Test is probably not the best tool to evaluate the impact of acute exercise interventions on attention. It seems plausible that use of a computerized test with a greater ability to precisely measure responses and the ability to introduce more randomness in the test stimuli could have yielded different results. 
In conclusion, we found no evidence to suggest that moderate aerobic or coordinative exercise has a beneficial effect on attention in graduate students. It is possible that these findings could have been influenced by exercise intensity or duration, and participants' perceptions of the physical activity interventions. There also seems to be increasing evidence to suggest that the $\mathrm{d} 2$ Test may not be well suited to assess attention in an acute exercise environment.

\section{CONFLICT OF INTEREST}

The authors confirm that this article content has no conflict of interest.

\section{ACKNOWLEDGEMENTS}

Declared none

\section{REFERENCES}

[1] Gomez-Pinilla F, Hillman C. The influence of exercise on cognitive abilities. Compr Physiol 2013; 3(1): 403-8.

[2] Hopkins ME, Davis FC, Vantieghem MR, Whalen PJ, Bucci DJ. Differential effects of acute and regular physical exercise on cognition and affect. Neuroscience 2012; 26; 215: 59-68.

[3] Nanda B, Balde J, Manjunatha S. The acute effects of a single bout of moderate-intensity aerobic exercise on cognitive functions in healthy adult males. J Clin Diagn Res 2013; 7(9): 1883-5.
[4] Tomporowski PD. Effects of acute bouts of exercise on cognition. Acta Psychol (Amst) 2003; 112(3): 297-324.

[5] Sibley BA, Etnier JL. The relationship between physical activity and cognition in children a meta-analysis. Pediat Exerc Sci 2003; 15: 243-56.

[6] Sousa DA. How the Brain Learns. 3rd ed. Thousand Oaks, CA: Corwin Press 2006.

[7] Bunce D, Flens E, Neiles K. How long can students pay attention in class? A study of student attention decline using clickers. J Chem Educ 2010; 87(12): 1438-43.

[8] Gao JH, Parsons LM, Bower JM, Xiong J, Li J, Fox PT. Cerebellum implicated in sensory acquisition and discrimination rather than motor control. Science 1996; 272(5261): 545-7.

[9] Courchesne E, Townsend J, Akshoomoff NA, et al. Impairment in shifting attention in autistic and cerebellar patients. Behav Neurosci 1994; 108(5): 848-65.

[10] Budde H, Voelcker-Rehage C, Pietrabyk-Kendziorra S, Ribeiro P, Tidow G. Acute coordinative exercise improves attentional performance in adolescents. Neurosci Lett 2008; 441(2): 219-23.

[11] American College of Sports Medicine. ACSM's Guidelines for Exercise Testing and Prescription. $9^{\text {th }}$ ed. Baltimore: Lippincott, Williams, \& Wilkins 2013.

[12] Brickenkamp R, Zillmer E. The d2 Test of Attention. Seattle, WA: Hogrefe and Huber Publishers 1998.

[13] Budde $\mathrm{H}$, Brunelli A, Machado S, et al. Intermittent maximal exercise improves attentional performance only in physically active students. Arch Med Res 2012; 43(2): 125-31.

[14] Gallotta MC, Guidetti L, Franciosi E, Emerenziani GP, Bonavolonta V, Baldari C. Effects of varying type of exertion on children's attention capacity. Med Sci Sports Exerc 2012; 44(3): 550-5.

[15] Palmer KK, Miller MW, Robinson LE. Acute exercise enhances preschoolers' ability to sustain attention. J Sport Exerc Psychol 2013; 35(4): 433-7.

Received: June 23, 2014

Revised: October 15, 2014

Accepted: October 21, 2014

(C) Bailey et al.; Licensee Bentham Open.

This is an open access article licensed under the terms of the Creative Commons Attribution Non-Commercial License (http://creativecommons.org/licenses/ by-nc/3.0/) which permits unrestricted, non-commercial use, distribution and reproduction in any medium, provided the work is properly cited. 